\title{
A New Approach for Event Detection and Feature Extraction for NILM
}

\author{
Nabil Amirach ${ }^{1,2}$, Bernard Xerri ${ }^{1,2}$, Bruno Borloz ${ }^{1,2}$, Claude Jauffret ${ }^{1,2}$ \\ ${ }^{1}$ Aix Marseille Université (CNRS, IM2NP, UMR 7334), 13397 Marseille, France \\ ${ }^{2}$ Université de Toulon (CNRS, IM2NP, UMR 7334), 83957 La Garde, France \\ amirach@univ-tln.fr
}

\begin{abstract}
The method proposed in this paper aims both to detect the events occurred on the overall current measurement and to estimate the features of the electric load underlying these events. This method is within the scope of the non-intrusive approach consisting in extracting information from the electric consumption only measured at the electrical switchboard.
\end{abstract}

Keywords - Non-intrusive load monitoring (NILM); event detection; feature extraction, estimation.

\section{INTRODUCTION}

Since last years, in order to save energy, the need to monitor the power consumption of residential and industrial processes has became an important problem.

The purpose of the smart grid is to have an accurate and reliable knowledge of a given electrical network in order to optimize the electricity consumption through the analysis of the overall current. A main issue is to decompose the total electrical consumption of a given grid in order to provide a detailed analysis of electrical consumption of each device present in the electrical network.

One can find two types of approaches: the intrusive ones which use one sensor for each appliance, and the non-intrusive load monitoring (NILM) one which aims at estimating the load consumption from a unique overall current and voltage measurement. This last problem is wildly addressed in the literature since the last 30 years [1-10].

NILM was first introduced by G.W. Hart [1] [2] where only the active power was considered. An appliance turning on or off is detected when the difference in the power consumption exceeds a predefined threshold. Then Hart proposed an improvement by using reactive power and harmonics in order to classify two loads with a same active power consumption.

Other solutions use the current signal instead of active and reactive power for event detection and feature extraction. In [10], the matrix pencil method estimates the parameters of a current modeled as a sum of exponentially damped sinusoids characterizing the loads.

Most approaches proposed in the literature are divided into four stages. Foremost the event detection and the feature extraction stage are processed. Then a classification stage uses the information provided by the preceding modules, in order to associate each event with the appliance which caused it. Finally, a last stage estimates the power consumption of each appliance.
In this study, we focus on the event detection and the feature extraction stages and the paper is organized as follows:

- in section II, we propose a new model of current matched to accurate transition detection,

- in section III, we develop a parameters estimation method for our new model,

- $\quad$ in section IV, we present some application examples.

\section{PROBLEM STATEMENT}

The only available information is the overall current and voltage measurement (respectively $\tilde{x}(t)$ and $\tilde{y}(t)$ ). One has chosen to modelize the voltage in steady state by:

$$
y(t)=\sum_{m=1}^{N_{f}} A_{m} \cos \left(2 \pi f_{m} t+\varphi_{m}\right)
$$

where $N_{f}$ is the number of frequencies $f_{m}$ with $0 \leq f_{m}<\frac{F e}{2}$. The amplitude and phase of the $m t h$ frequency component are $A_{m} \geq 0$ and $\varphi_{m} \in[0,2 \pi)$. Let's define the vectors $f=$ $\left[\begin{array}{lll}f_{1} & \cdots & f_{N_{f}}\end{array}\right]^{T}, \quad A=\left[\begin{array}{lll}A_{1} & \cdots & A_{N_{f}}\end{array}\right]^{T} \quad$ and $\quad \varphi=\left[\begin{array}{lll}\varphi_{1} & \cdots & \varphi_{N_{f}}\end{array}\right]^{T}$, where $[.]^{T}$ denotes the transposition operator.

The events occur on the current signal; we will try to detect and extract the features from $\tilde{x}(t)$ and $\tilde{y}(t)$. Considering Kirchhoff's law, the overall current is composed by the sum of the currents consumed by each system in use which contains information about frequencies. The model of the current consumed by a linear load in steady state is similar to (1).

Unlike to the matrix pencil method which estimates the frequencies $f$ and their number $N_{f}$ [11] [12], they are here supposed to be known.

In previous studies, the current was modelized by a sum of damped sinusoids. However, the current measured on a grid is made of a sequence of events (transitions) due to the switching on and off of devices. Figure 1 shows the different scenarios that may be encountered during processing (different cases of observing window):

1) This case represents the current on initial steady state, before that another load turns on.

2) Here, there is a "positive" transition in the observation window. Before the transition is the current consumed 
by the already running loads, and after, there is the sum of the previous current and the current consumed by the load which has switched on.

3) In this and the fifth cases, there is no transition. We observe the current consumed by the overall system in steady state.

4) Now we observe a "negative" transition. A load is turned off.

If the model previously defined is used for the cases 2) and 4 ), it will estimate the parameters of an "average" signal of the signals before and after the transition. The model above is not matched to these scenarios: it must take into account the existence of a transition in the observation window.

Figure 2 shows the case of an observation window of duration $T$ containing a transition at time $T_{1}$ (e.g. case 2)). $T_{2}$ is the duration of the signal added by the load which turned on. This transition is in fact related to a system that turns on or turns off. From this window, we must detect the transition instant and characterize the load which is the source of this transition. When a transition occurs, the model (eq (1)) must be rewritten as two sums of cosine: it is simplified as a single sum of cosine when there is no transition. The first sum represents the overall system before the transition, and the second one the system which was turned on.

In the observing window, the signal is modelized as:

$$
\begin{aligned}
x(t)=\sum_{m=1}^{N_{f}} A_{1, m} \cos \left(2 \pi f_{m} t+\varphi_{1, m}\right) \cdot \Pi_{T}(t) & \\
& +\sum_{m=1}^{N_{f}} A_{2, m} \cos \left(2 \pi f_{m} t+\varphi_{2, m}\right) \cdot \Pi_{T_{2}}\left(t-T_{1}\right)
\end{aligned}
$$

with $A_{i}=\left[A_{i, 1} \cdots A_{i, N_{f}}\right]^{T}$ and $\varphi_{i}=\left[\varphi_{i, 1} \cdots \varphi_{i, N_{f}}\right]^{T}: i=1$ means before the transition, and $i=2$ represent the load which causes the transition. $\Pi_{T_{2}}\left(t-T_{1}\right)$ denotes the rectangular window function of length $T_{2}$ starting in $T_{1}\left(T=T_{1}+T_{2}\right)$.

Comparing to Figure 2, we can see that the model takes into account all possible scenarios. It corresponds to the cases of an observing window with both a transition $\left(0<T_{2}<T\right)$ and without a transition $\left(T_{2}=0\right.$ or $\left.T_{2}=T\right)$.

The theoretical development above is expressed in the continuous time domain. However we work with discrete signals. So let us transpose these expressions in the discrete time domain.

We define $N$ the number of samples in the observing window, $N_{1}$ the sample number where the transition begins and $N_{2}=N-N_{1}+1$ the number of samples of the added signal. The duration of the observing window and others are defined by (with $\Delta t$ the sampling period):

$$
T=(N-1) \cdot \Delta t, T_{1}=\left(N_{1}-1\right) \cdot \Delta t, T_{2}=\left(N_{2}-1\right) \cdot \Delta t
$$

The unknown parameters are $\mathrm{A}_{1, \mathrm{~m}}, \varphi_{1, \mathrm{~m}}, \mathrm{~A}_{2, \mathrm{~m}}, \varphi_{2, \mathrm{~m}}$ and $\mathrm{N}_{2}$.

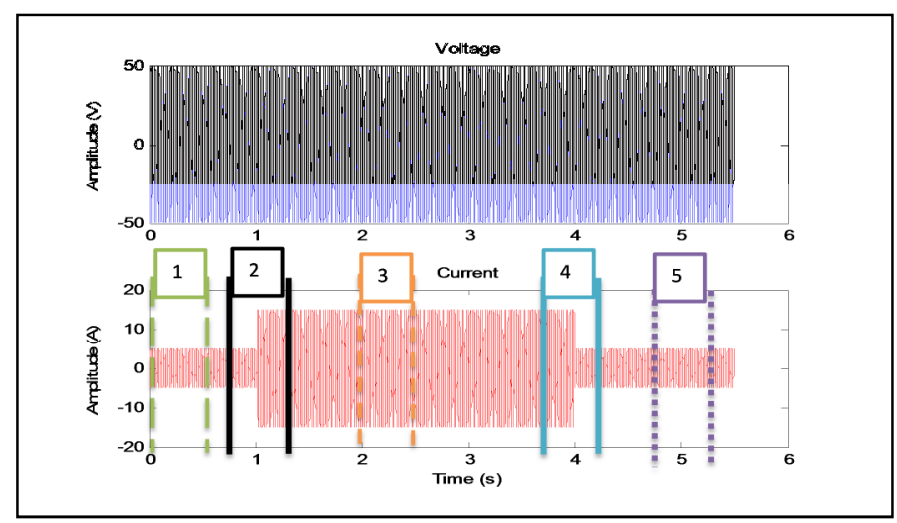

Figure 1. Example of voltage and current (cases 1) to 5))

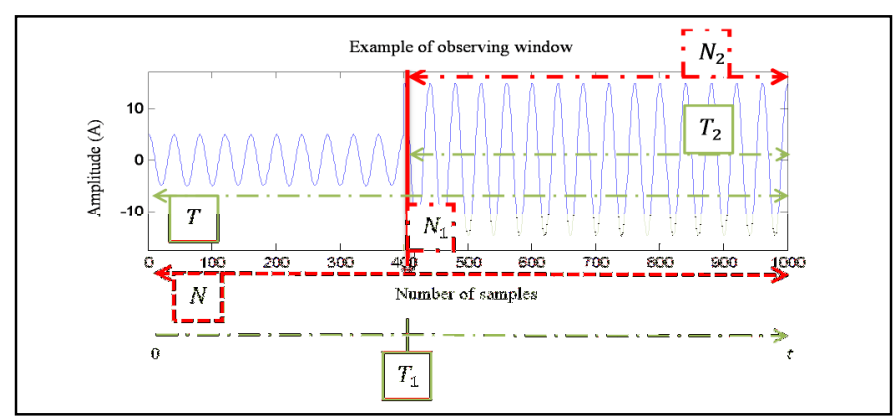

Figure 2. Example of observing window (case 2))

\section{EVENT DETECTION AND FEATURE EXTRACTION}

Let us develop a method to estimate these parameters. And characterize the load in the frequency domain. Using the discrete Fourier transform, the model is more towable to estimate $N_{2}$. Thus, one obtains the given frequency model:

$$
\begin{aligned}
& X(f) \\
& =\sum_{m=1}^{N_{f}}\left\{\frac{A_{1, m}}{2} e^{j \varphi_{1, m}} \cdot \frac{\sin \left(\pi\left(f-f_{m}\right) T\right)}{\sin \left(\pi\left(f-f_{m}\right) \Delta t\right)} \cdot e^{-j \pi\left(f-f_{m}\right) T}\right. \\
& +\frac{A_{1, m}}{2} e^{-j \varphi_{1, m}} \cdot \frac{\sin \left(\pi\left(f+f_{m}\right) T\right)}{\sin \left(\pi\left(f+f_{m}\right) \Delta t\right)} e^{-j \pi\left(f+f_{m}\right) T} \\
& +\frac{A_{2, m}}{2} e^{j \varphi_{2, m}} \cdot \frac{\sin \left(\pi\left(f-f_{m}\right) T_{2}\right)}{\sin \left(\pi\left(f-f_{m}\right) \Delta t\right)} \cdot e^{-2 j \pi\left(f-f_{m}\right)\left(\frac{T_{2}}{2}+T_{1}\right)} \\
& \left.+\frac{A_{2, m}}{2} e^{-j \varphi_{2, m}} \cdot \frac{\sin \left(\pi\left(f+f_{m}\right) T_{2}\right)}{\sin \left(\pi\left(f+f_{m}\right) \Delta t\right)} \cdot e^{-2 j \pi\left(f+f_{m}\right)\left(\frac{T_{2}}{2}+T_{1}\right)}\right\}
\end{aligned}
$$

During the steady state preceding the transition, one can obtain an estimation of the vectors $A_{1}$ and $\varphi_{1}$ (using Least Squares method). That's why these vectors will be considered to be known. And we know that $A_{2}=0$ and $\varphi_{2}=0$. Knowing $\widehat{\mathrm{A}}_{1}$ and $\widehat{\varphi}_{1}$, the current measured before the transition can be removed by calculating the error between the measured signal in the observing window, and the signal estimate constructed with $\widehat{\mathrm{A}}_{1}$ and $\widehat{\varphi}_{1}$. The residue obtained, denoted $\widetilde{\mathrm{X}}_{2}(\mathrm{f})$ is in fact the current consumed by the system which turns on. Now, using $\widetilde{X}_{2}(f)$, we can characterize this system by estimating the parameters of its model denoted $\mathrm{X}_{2}(\mathrm{f})$. Therefore, using

$$
\mathcal{F}\left(\mathrm{N}_{2}, \mathrm{f}\right)=\frac{\sin \left(\mathrm{N}_{2} \cdot \pi \mathrm{f} \Delta \mathrm{t}\right)}{\sin (\pi \mathrm{f} \Delta \mathrm{t})} \cdot \mathrm{e}^{-\mathrm{j} \pi \mathrm{f}\left(2 \mathrm{~N}-1-\mathrm{N}_{2}\right) \Delta \mathrm{t}}
$$


one has

$$
\begin{aligned}
X_{2}(f)=\sum_{m=1}^{N_{f}} \frac{A_{2, m}}{2} & e^{j \varphi_{2, m}} \cdot \mathcal{F}\left(N_{2}, f-f_{m}\right) \\
& +\frac{A_{2, m}}{2} e^{-j \varphi_{2, m}} \cdot \mathcal{F}\left(N_{2}, f+f_{m}\right)
\end{aligned}
$$

One estimates $\mathrm{N}_{2}, \mathrm{~A}_{2}$ and $\varphi_{2}$ from $\mathrm{X}_{2}$ (f) using a least squares (LS) criterion:

$$
\mathrm{C}\left(\mathrm{N}_{2}, \mathrm{~A}_{2}, \varphi_{2}\right)=\sum_{\mathrm{k}=1}^{\mathrm{N}}\left|\tilde{X}_{2}\left(f_{k}\right)-X_{2}\left(f_{k}\right)\right|^{2}
$$

where $\mathrm{f}_{\mathrm{k}}=(\mathrm{k}-1) \Delta \mathrm{f}, \Delta \mathrm{f}=\frac{\mathrm{F}_{\mathrm{s}}}{\mathrm{N}}=\frac{1}{N \cdot \Delta t}$.

The estimate in the least squares sense is the vector $\left(\widehat{N}_{2}, \hat{A}_{2}, \hat{\varphi}_{2}\right)$ that minimizes $C\left(N_{2}, A_{2}, \varphi_{2}\right)$. We note that:

$X_{2}\left(f_{k}\right)=\frac{1}{2} \cdot\left[\begin{array}{c}\mathcal{F}\left(N_{2}, f_{k}-f_{1}\right) \\ \mathcal{F}\left(N_{2}, f_{k}+f_{1}\right) \\ \vdots \\ \mathcal{F}\left(N_{2}, f_{k}-f_{N_{f}}\right) \\ \mathcal{F}\left(N_{2}, f_{k}+f_{N_{f}}\right)\end{array}\right]^{T} \cdot\left[\begin{array}{c}A_{2,1} e^{j \varphi_{2,1}} \\ A_{2,1} e^{-j \varphi_{2,1}} \\ \vdots \\ A_{2, N_{f}} e^{j \varphi_{2, N_{f}}} \\ A_{2, N_{f}} e^{-j \varphi_{2, N_{f}}}\end{array}\right]=B_{k}\left(N_{2}\right) \cdot \theta$

The minimization of $\mathrm{C}\left(\mathrm{N}_{2}, \mathrm{~A}_{2}, \varphi_{2}\right)$ is performed using the two following stages.

Stage 1: set $\mathrm{N}_{2}$, and compute the vector $\hat{\theta}\left(\mathrm{N}_{2}\right)$ that minimizes the following criterion

$$
C_{N_{2}}(\theta)=\sum_{k=1}^{N}\left|\tilde{X}_{2}\left(f_{k}\right)-B_{k}\left(N_{2}\right) \cdot \theta\right|^{2}
$$

This minimization is performed by:

$$
\hat{\theta}\left(N_{2}\right)=\left(\sum_{l} B_{l}^{H}\left(N_{2}\right) B_{l}\left(N_{2}\right)\right)^{-1} \sum_{n} B_{n}^{H}\left(N_{2}\right) \tilde{X}_{2}\left(f_{n}\right)
$$

Note: Here ${ }^{H}$ represents the conjugate transpose of a vector or a matrix.

Stage 2: search $\widehat{N}_{2}$ which minimizes the following criterion

$$
C\left(N_{2}\right)=\sum_{k=1}^{N}\left|\tilde{X}_{2}\left(f_{k}\right)-B_{k}\left(N_{2}\right) \cdot \hat{\theta}\left(N_{2}\right)\right|^{2}
$$

Then, we select the estimate $\hat{\theta}$ corresponding to this value of $\widehat{\mathrm{N}}_{2}$. Finally, calculating the modulus and argument of $\hat{\theta}$ provides the estimated amplitudes and phases $\left(\widehat{\mathrm{A}}_{2}\right.$ and $\left.\widehat{\varphi}_{2}\right)$.

The estimation is also performed on, the amplitudes and phases of the voltage in order to calculate the admittance (or impedance) associated with each frequency. The voltage is assumed without transition. But, it may have some variations when an event occurs on the current. However, we do not have to estimate the transition instant. This allows to enhance the information vector. Unlike to most of the methods found in the literature, it does not only work on the fundamental frequency. At each transition, the features extracted are:
- the instant and duration of the transitions

- the frequencies present in the current consumed by loads that turn on,

- the admittances associated with each of these frequencies.

These features are then processed by the classification algorithm where is compared the information of each transition. If two transitions have the same frequencies, and if, for each frequency, the modulus of the admittance is the same and the phases are shifted by $180^{\circ}$, then these transitions correspond to a load which has been turned on and off.

\section{RESUlTS}

In this section, one presents an application on simulated signals and another one on real signals.

A voltage $y$ is created containing following harmonics $\mathrm{J}=\{1,3,5\}$, so:

$$
y\left(t_{k}\right)=\sum_{j \in J} U_{1, j} \cos \left(2 \pi f_{j} t_{k}+\psi_{1, j}\right)
$$

and $\mathrm{f}=[50 \mathrm{~Hz}, 150 \mathrm{~Hz}, 250 \mathrm{~Hz}]^{\mathrm{T}}$

$$
U_{1}=[50,1,0.5], \psi_{1}=[90,60,45]
$$

Then three currents are simulated and noise free, each representing the current consumed by a load. These currents compose the overall current $x$ :

$$
x\left(t_{k}\right)=x_{1}\left(t_{k}\right)+x_{2}\left(t_{n}\right)+x_{3}\left(t_{m}\right)
$$

where:

$$
\begin{gathered}
\mathrm{x}_{\mathrm{i}}\left(\mathrm{t}_{\mathrm{k}}\right)=\sum_{\mathrm{j} \in \mathrm{J}} \mathrm{I}_{\mathrm{i}, \mathrm{j}} \cos \left(2 \pi \mathrm{f}_{\mathrm{j}} \mathrm{t}_{\mathrm{k}}+\varphi_{\mathrm{i}, \mathrm{j}}\right) \\
t_{k}=(k-1) \Delta t, \Delta \mathrm{t}=\frac{1}{\mathrm{~F}_{\mathrm{s}}}, \mathrm{F}_{\mathrm{s}}=2 \mathrm{kHz} \text { with } D_{\mathrm{i}}(1)<k<D_{\mathrm{i}}(2) \\
\mathrm{D}_{1}=[1 ; 11000], \mathrm{D}_{2}=[2036 ; 6321], \mathrm{D}_{3}=[4012 ; 9012] \\
\mathrm{I}_{1}=[30,2,0.25], \varphi_{1}=[30,0,90] \\
\mathrm{I}_{2}=[42,3,1], \varphi_{2}=[90,36,-90] \\
\mathrm{I}_{3}=[12,1,0.5], \varphi_{3}=[30,0,90]
\end{gathered}
$$

Four transitions underlied by two loads are simulated (consuming $\mathrm{x}_{2}$ and $\mathrm{x}_{3}$ ). The first one turns on at $1.018 \mathrm{~s}$ and turns off at $3.1605 \mathrm{~s}$. The second one turns on at $2.006 \mathrm{~s}$ and turns off at 4.506s. Each simulated admittance modulus and phase are calculated as follow:

- $\quad$ the modulus is the ratio of $I$ and $U$

- $\quad$ the argument is the difference between $\varphi$ and $\varphi_{\mathrm{i}}$

and are given here:

$$
\begin{aligned}
& M_{2}=[0.84,3,2], P_{2}=[0,-24,-135] \\
& M_{3}=[0.24,1,1], P_{3}=[-60,-60,45]
\end{aligned}
$$

Figure 3 show the result of the detection algorithm. It perfectly detects these transitions. From table I which contain 
numerical results, we note that the first and third transitions have the same amplitudes. In addition estimates of the third transition phases are shifted by $180^{\circ}$ from the first transition. It is the same for the second and fourth transitions. The extracted information allows us to say that the first and third transitions are caused by the same load (turning on and off). And the two other are caused by a second load.

Table II contains the result using our algorithm on real measurements (realized at the University of Toulon). These are the voltage and the current measured across an appliance given as resistor.

The extracted information corroborates the results obtained on simulated signals. Indeed we can see that the modulus is the same for both the first and second transition. And the phases are shifted by $180^{\circ}$. So these transitions are due to the same load switching on and off. From table II, the phase difference between the current and the voltage is almost equal to $0^{\circ}$ for all frequencies, except for $150 \mathrm{~Hz}$. The admittance modulus of this load is approximately equal to 0.24 except for $150 \mathrm{~Hz}$. Then, it appears that the detected load is a resistor with impedance about $4 \Omega$ and with an unexpected behaviour around $150 \mathrm{~Hz}$ perhaps due to a non-linearity.

\section{CONCLUSION}

The method proposed in this paper allows to detect each transition and extract its date and duration, and then to estimate the frequencies parameters (amplitudes and phases). Moreover, other information is the number of frequencies (often harmonics) which is different depending on the appliance. Thus the estimated features can be used to improve the classification of the appliances detected in the network.

Studies have been made on measurements disturbed with additive random noise. Or when there exists frequencies which are not taken into account. And it appears that the results provided by our algorithm are still good.

\section{ACKNOWLEDGMENT}

The authors would like to thank the Provence Alpes Côte d'Azur (PACA) region and the company Qualisteo for their financial support.

\section{REFERENCES}

[1] G. Hart, "Residential energy monitoring and computerized surveillance via utility power flows", IEEE Technology and Society, pp. 12-16, June 1989.

[2] G.W. Hart, "Nonintrusive Appliance Load Monitoring,", Proceedings of the IEEE, December 1992, pp. 1870-1891.

[3] Norford, L. K. and S. B. Leeb. 1996. "Nonintrusive Electrical Load Monitoring." Energy and Buildings, Vol. 24, pp. 51-64.

[4] Leeb, S. B., S. R. Shaw, and J. L. Kirtley, Jr. 1995. "Transient Event Detection in Spectral Envelope Estimates for Noninstrusive Load Monitoring." IEEE Transactions on Power Delivery Vol. 10 No. 3, pp. 1200-1210.

[5] Cole, A. I. and Albicki, A. "Algorithm for non-intrusive identification of residential appliances", Proc. of the 1998 IEEE International Symposium on Circuits and Systems (ISCAS '98), Vol. 3, May 31- June 3, 1998, pp.338- 341 .

[6] Laughman, C., Lee, K., Cox, R., Shaw, S., Leeb, S., Norford, L., and Armstrong, P. "Power Signature Analysis," IEEE Power and Energy Magazine, V. 1(2), Mar-Apr 2003, Pages 56-63.

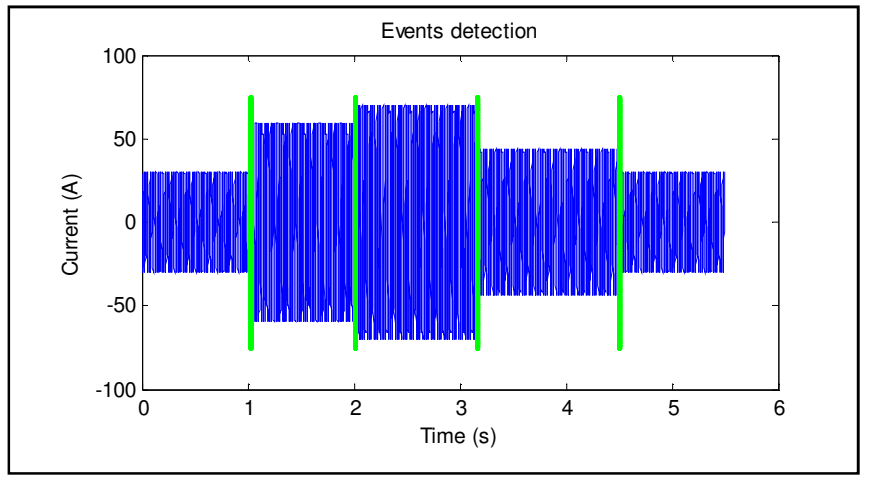

Figure 3. Current signal and transition instant detected

TABLE I. FEATURES EXTRACTION RESULT

\begin{tabular}{|c|c|c|c|c|c|}
\hline \multirow{2}{*}{$\begin{array}{c}\text { Harmonic } \\
\text { frequencies (Hz) }\end{array}$} & \multicolumn{4}{|c|}{ Estimated transition instant: $\widehat{\boldsymbol{N}}_{\mathbf{1}}$} \\
\cline { 3 - 6 } & $\mathbf{2 0 3 6}$ & $\mathbf{4 0 1 2}$ & $\mathbf{6 3 2 2}$ & $\mathbf{9 0 1 3}$ \\
\cline { 3 - 6 } 50 & Modulus & 0.84 & 0.24 & 0.84 & 0.24 \\
\cline { 2 - 6 } & Phase $\left(^{\circ}\right)$ & 0 & -60 & -180 & 120 \\
\hline \multirow{2}{*}{150} & Modulus & 3 & 1 & 3 & 1 \\
\cline { 2 - 6 } & Phase $\left(^{\circ}\right)$ & -24 & -60 & 156 & 120 \\
\hline \multirow{2}{*}{250} & Modulus & 2 & 1 & 2 & 1 \\
\cline { 2 - 6 } & Phase $\left(^{\circ}\right)$ & -135 & 45 & 45 & -135 \\
\hline
\end{tabular}

TABLE II. RESULTS ON REAL SIGNALS

\begin{tabular}{|c|c|c|c|}
\hline \multirow{3}{*}{\multicolumn{2}{|c|}{$\begin{array}{c}\text { Harmonic } \\
\text { frequencies }(\mathrm{Hz})\end{array}$}} & \multicolumn{2}{|c|}{ Estimated transition instant: $\widehat{N}_{1}$} \\
\hline & & 3183 & 8026 \\
\hline & & \multicolumn{2}{|c|}{ Estimated admittances } \\
\hline \multirow{2}{*}{50} & Modulus & 0.246 & 0.239 \\
\hline & Phase $\left(^{\circ}\right)$ & -0.253 & 179.752 \\
\hline \multirow{2}{*}{150} & Modulus & 0.382 & 0.386 \\
\hline & Phase $\left({ }^{\circ}\right)$ & 47.9 & -132.821 \\
\hline \multirow{2}{*}{350} & Modulus & 0.249 & 0.224 \\
\hline & Phase $\left({ }^{\circ}\right)$ & -0.849 & 179.176 \\
\hline
\end{tabular}

[7] Leeb S. B., Shaw S. R., Kirtly J. L., Jr., "Transient event detection in spectral envelop estimates for nonintrusive load monitoring," IEEE Trans. Power Deliv., Vol. 10, No. 3, 1995, pp. 1200-1210.

[8] M. El Guedri, G D’Urso, C. Lajaunie and G. Fleury "Time-frequency characterisation for electric load monitoring," European Signal Processing Conference 2009 (EUSIPCO) : Glasgow-Scotland, 2009

[9] M. El Guedri "Caractérisation aveugle de la courbe de charge électrique: Détection, classification et estimation des usages dans les secteursz résidentiel et tertiaire", Thesis, Paris-Sud 11 University, november 2009.

[10] N. Hala "Méthode d'identification et de classification de la consommation d'énergie par usages en vue de l'intégration dans un compteur d'énergie électrique", Thesis, Blaise-Pascal-Clermont II University, December 2009

[11] Y. Hua et T. K. Sarkar "Matrix Pencil Method for Estimating Parameters of Exponentially Damped/Undamped Sinusoids in Noise", IEEE Transaction on Acoustics, Speech and Signal Processing, Vol. 38, No. 5, May 1990.

[12] T. K. Sarkar and O. Pereira "Using the Matrix Pencil Method to Estimate the Parameters of a Sum of Complex Exponentials", IEEE Antennas and Propagation Magazine, Vol. 37, No. 1, February 1995. 
